\title{
DIFFRACTIVE JET PRODUCTION IN DIS - TESTING QCD FACTORISATION
}

\author{
F.-P. SCHILLING [H1 COLLABORATION] \\ DESY, Notkestr. 85, D-22603 Hamburg, Germany \\ E-mail:fpschill@mail.desy.de
}

\begin{abstract}
A measurement 4 . 2 is presented of diffractive dijet production in deep-inelastic scattering (DIS) interactions at HERA of the type $e p \rightarrow e X Y$, where the $X$ system is separated by a large rapidity gap from a low-mass leading baryonic system $Y$. The data are consistent with QCD factorisation in diffractive DIS and yield tight constraints on both shape and normalisation of the diffractive gluon distribution. The data are able to distinguish between various models for diffractive DIS and are in agreement with a 2 -gluon exchange calculation at small $x_{\mathbb{P}}$ values.
\end{abstract}

\section{Overview}

At HERA, colour singlet exchange or diffractive processes are studied in deepinelastic ep scattering (DIS), where the exchanged photon with virtuality $Q^{2}$ provides a probe to determine the QCD (i.e. quark and gluon) structure of diffractive exchange. In $\mathrm{B}$, it was proven that QCD hard scattering factorisation is valid in diffractive DIS, so that diffractive parton distributions $p_{i}^{D}$ in the proton can be defined as quasi-universal objects. The hypothesis of a factorising $x_{\mathbb{P}}$ dependence (Regge factorisation) is often used in addition.

Measurements of inclusive diffractive DIS in terms of the diffractive structure function $F_{2}^{D(3)}\left(x_{\mathbb{P}}, \beta, Q^{2}\right)$ mainly constrain the diffractive quark distribution. By contrast, diffractive dijet production is directly sensitive to the gluon distribution $g^{D}\left(z, \mu^{2}\right)$ (Fig. 1), which can be inferred only indirectly from the scaling violations of $F_{2}^{D(3)}$. QCD factorisation can be tested by predicting the dijet cross sections using the pdf's extracted from $F_{2}^{D(3)}$.

Furthermore, the predictions of a variety of phenomenological models for diffractive DIS such as soft colour neutralisation or 2-gluon exchange can be confronted with the dijet cross sections.

\section{Data Selection and Cross Section Measurement}

The data sample corresponds to an integrated luminosity of $\mathcal{L}=18.0 \mathrm{pb}^{-1}$ and was obtained with the H1 detector at HERA. Dijet events were identified using the CDF cone algorithm and diffractive events were selected by the requirement of a large rapidity gap in the outgoing proton direction. The 

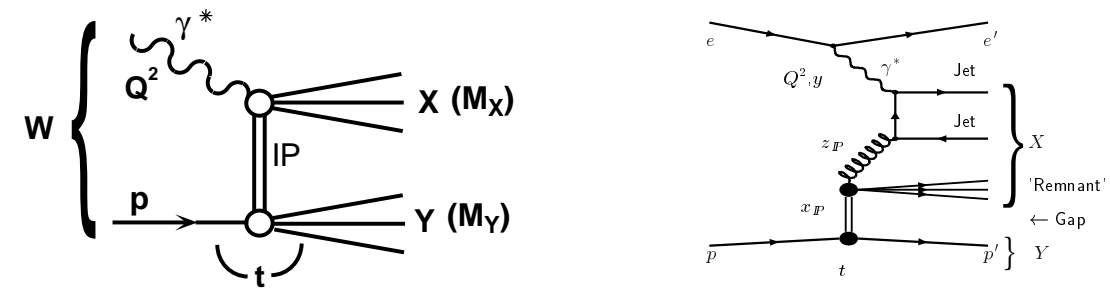

Figure 1. Inclusive diffractive scattering at HERA (left) and diffractive dijet production (right), viewed in a partonic picture.

kinematic range of the measurement is $4<Q^{2}<80 \mathrm{GeV}^{2}, p_{T, \text { jet }}^{*}>4 \mathrm{GeV}$, $x_{\mathbb{P}}<0.05, M_{Y}<1.6 \mathrm{GeV}$ and $|t|<1.0 \mathrm{GeV}^{2}$. The cross sections were corrected for detector and QED radiative effects and the systematic uncertainties, which dominate the total errors, were carefully evaluated.

\section{Diffractive Parton Distributions}

Parton distributions for the diffractive exchangef were extracted from DGLAP QCD fits to $F_{2}^{D(3)}\left(x_{\mathbb{P}}, \beta, Q^{2}\right)$ in $⿴$. The parton distributions were found to be dominated by gluons $(80-90 \%$ of the exchange momentum).

If these parton distributions, which evolve according to the DGLAP equations, are used to predict the diffractive dijet cross sections, a very good agreement is obtained (Fig. 2). Fig. 3 shows the measurement of the dijet cross section as a function of $z_{\mathbb{P}}^{(\text {jets })}$, an estimator for the parton momentum fraction of the diffractive exchange which enters the hard process (Fig. 1 1 right). A very good agreement in shape and normalisation is obtained if the fit 2 parton distributions from $t$ are used. The fit 3 parameterisation, in which the gluon distribution is peaked towards high $z_{\mathbb{P}}$ values, is disfavoured 9 . Using different factorisation scales $\left(\mu^{2}=Q^{2}+p_{T}^{2}\right.$ in Fig. 3a $\mathrm{a}, \mu^{2}=p_{T}^{2}$ in Fig. Bb $\left.\mathrm{b}\right)$ or including a resolved virtual photon contribution (Fig. Ba) in the model prediction does not alter these conclusions. The dijet data thus strongly support the validity of QCD factorisation in diffractive DIS and give tight constraints on the diffractive gluon distribution in both shape and normalisation.

The measured $z_{\mathbb{P}}^{(\text {jets })}$ cross sections in bins of the scale $\mu^{2}=Q^{2}+p_{T}^{2}$ (Fig. 国a) are in good agreement with the prediction based on a DGLAP evo-

$a$ The assumption of Regge factorisation was found to be compatible with the data.

${ }^{b}$ The corresponding gluon distributions are shown above the cross sections. 

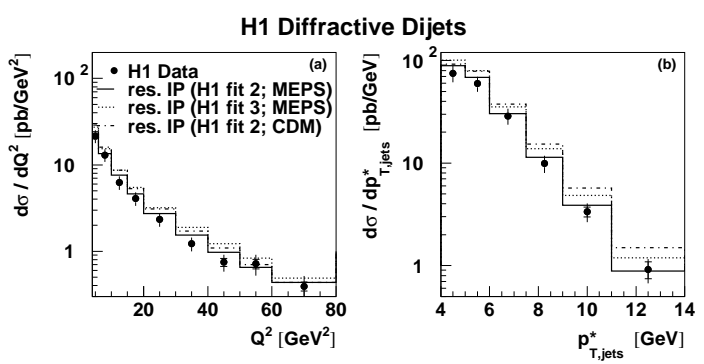

Figure 2. Diffractive dijet cross sections as a function of $(a) Q^{2}$ and $(b) p_{T, j e t s}^{*}$.

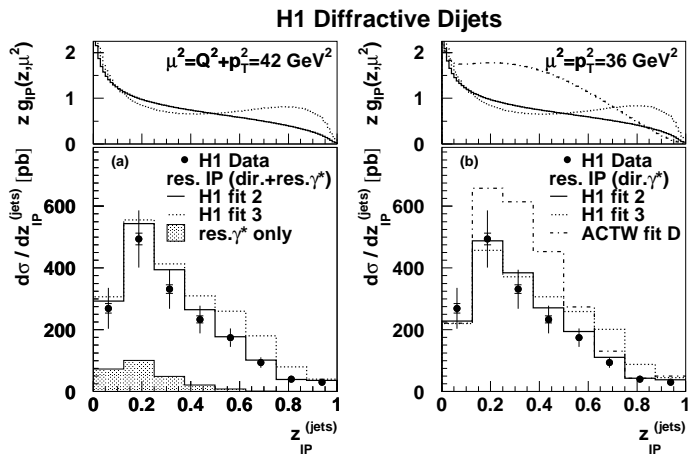

Figure 3. Diffractive dijet cross sections as a function of $z_{\mathbb{P}}^{(j e t s)}$. In $(a)$ and $(b)$, the same data are compared with different model predictions (see text).

lution of the diffractive parton distributions. The $z_{\mathbb{P}}^{(\text {jets })}$ cross sections in bins of $x_{\mathbb{P}}$ (Fig. (1) demonstrate consistency with Regge factorisation.

In a Regge framework, the energy dependence of the cross section is determined in terms of an effective pomeron intercept $\alpha_{\mathbb{P}}(0)=1.17 \pm 0.07$ (stat. +syst.) from the $x_{\mathbb{P}}$ cross section (Fig. Fa), consistent with the result from $\mathrm{t}$. The cross section as a function of $\beta$ is shown in Fig. Fb.

\section{Soft Colour Neutralisation Models}

In Fig. 6, the cross sections are compared with models based on the ideas of soft colour neutralisation to produce large rapidity gaps. These are the 

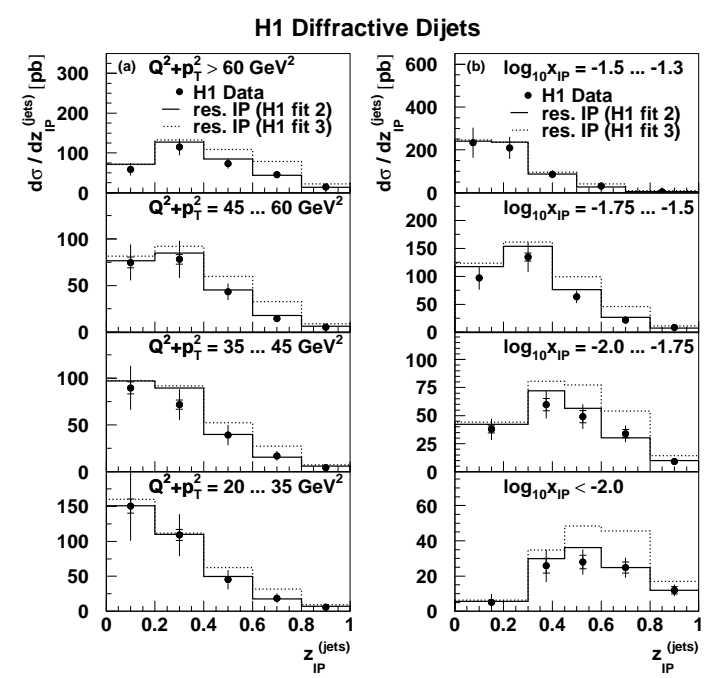

Figure 4. Diffractive dijet cross sections as a function of $z_{\mathbb{P}}^{(j e t s)}$ (a) in four bins of $\mu^{2}=$ $Q^{2}+p_{T}^{2}$ and (b) in four bins of $x_{\mathbb{P}}$.

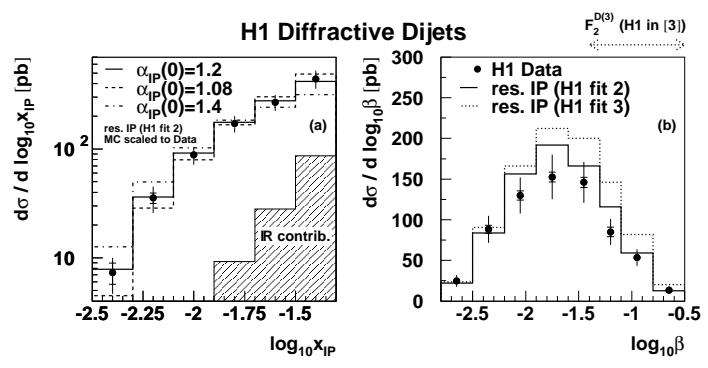

Figure 5. Diffractive dijet cross sections as a function of (a) $\log _{10} x_{\mathbb{P}}$ and (b) $\log _{10} \beta$.

original version of the 'soft colour interactions' model (SCI) 月, the improved version of SCI based on a generalised area law 6 and the 'semi-classical model' 6. The original SCI and the semi-classical models give good descriptions of the differential distributions. However, none of these models is yet able to simultaneously reproduce shapes and normalisations of the dijet cross sections. 

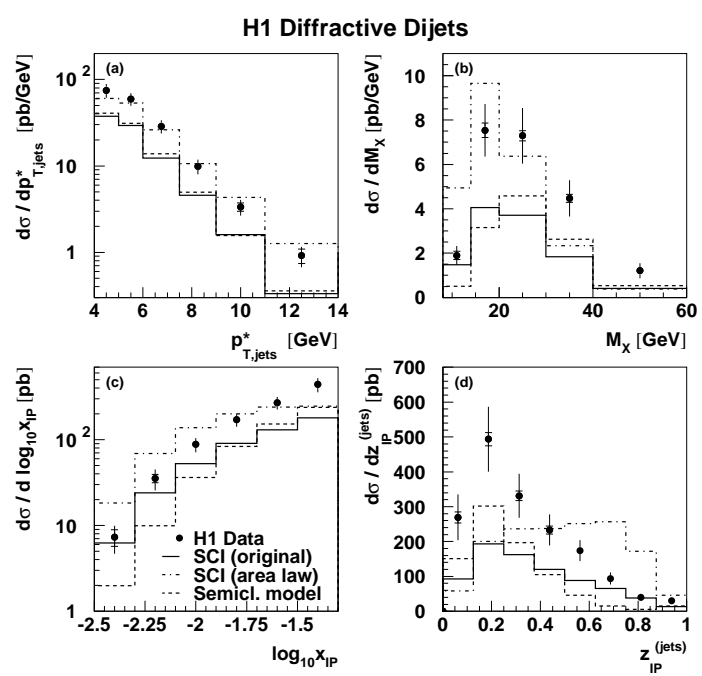

Figure 6. Diffractive dijet cross sections, compared with the predictions of soft colour neutralisation models (see text).

\section{Colour Dipole and 2-gluon Exchange Models}

Models for diffractive DIS based on the diffractive scattering of $q \bar{q}$ or $q \bar{q} g$ photon fluctuations off the proton by 2 -gluon exchange are confronted with the data in Fig. 目 for the limited kinematic range of $x_{\mathbb{P}}<0.01$, where contributions from quark exchange can be neglected. The 'saturation model' 8, which takes only $k_{T}$-ordered configurations of the final state partons into account, reproduces the shapes of the differential distributions, but underestimates the cross sections by a factor of two. The model of Bartels et al. $\mathrm{g}$, in which also non- $k_{T}$-ordered configurations are taken into account, is found to be in reasonable agreement with the data if a free parameter $p_{T, g}^{\text {cut }}$ is fixed to $1.5 \mathrm{GeV}$.

\section{Conclusions}

Diffractive dijet production has been shown to be a powerful tool to gain insight into the underlying QCD dynamics of diffraction, in particular the

${ }^{c} p_{T, g}^{\text {cut }}$ corresponds to the minimum $p_{T}$ of the final state gluon in the case of $q \bar{q} g$ production. 

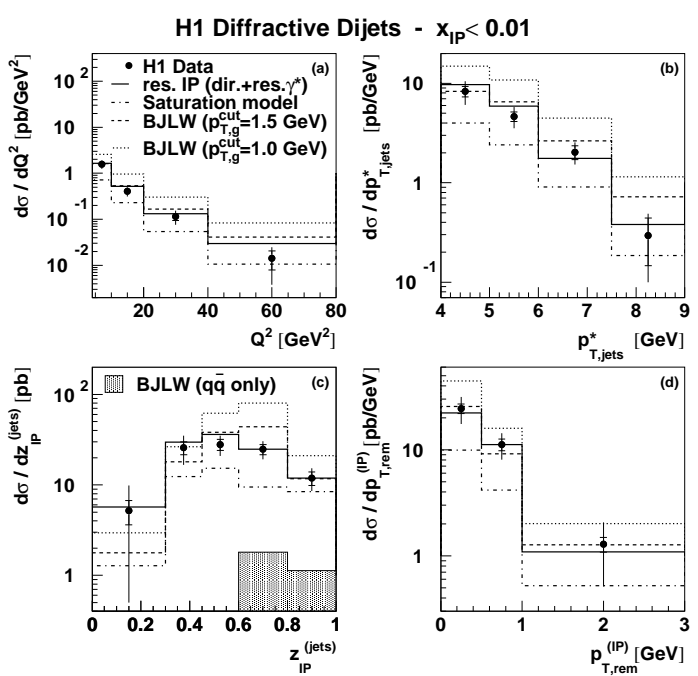

Figure 7. Diffractive dijet cross sections for $x_{\mathbb{P}}<0.01$, compared with the predictions of 2-gluon exchange models (see text).

role of gluons. Factorisable, gluon-dominated diffractive parton distributions successfully describe diffractive jet production and inclusive diffraction in DIS at the same time, in agreement with QCD factorisation.

\section{References}

1. H1 Collaboration, C. Adloff et al., Eur. Phys. J. C 20 (2001) 1,29.

2. F.-P. Schilling, Ph.D. thesis, Univ. Heidelberg (2001), DESY-THESIS2001-010.

3. J. Collins, Phys. Rev. D 57 (1998) 3051, err.-ibid. D 61 (2000) 19902.

4. H1 Collaboration, C. Adloff et al., Z. Phys. C 76 (1997) 613.

5. A. Edin, G. Ingelman, J. Rathsman, Phys. Lett. B 366 (1996) 371.

6. J. Rathsman, Phys. Lett. B 452 (1999) 364.

7. W. Buchmüller, T. Gehrmann, A. Hebecker, Nucl. Phys. B 537 (1999) 477.

8. K. Golec-Biernat, M. Wüsthoff, Phys. Rev. D 60 (1999) 114023.

9. J. Bartels, H. Lotter, M. Wüsthoff, Phys. Lett. B 379 (1996) 239;

J. Bartels, H. Jung, M. Wüsthoff, Eur. Phys. J. C 11 (1999) 111. 